\title{
Generalized Ostrowski-type inequalities involving second derivatives via the Katugampola fractional integrals
}

\section{Seth Kermausuor}

Department of Mathematics and Computer Science, Alabama State University, Montgomery, AL 36101, U. S. A.

\begin{abstract}
In this paper, we provide some Ostrowski-type integral inequalities for functions whose second derivatives belongs to the Lebesgue $\mathrm{L}_{\mathrm{q}}$ spaces using the Katugampola fractional integrals. We also introduced some new inequalities of Ostrowski-type for functions whose second derivatives in absolute value at some powers are strongly $(\mathrm{s}, \mathrm{m})$-convex with modulus $\mu \geqslant 0$ (in the second sense). Our results are generalizations of some earlier results in the literature.
\end{abstract}

Keywords: Ostrowski inequality, convex functions, strongly $(\mathrm{s}, \mathrm{m})$-convex functions, Riemann-Liouville fractional integrals, Hadamard fractional integrals, Katugampola fractional integrals, Hölder's inequality, power mean inequality.

2010 MSC: 26A33, 26A51, 26D10, 26D15.

(C)2019 All rights reserved.

\section{Introduction}

Let $I$ be an interval $\mathbb{R}$. A function $f: I \rightarrow \mathbb{R}$ is said to be convex if

$$
f(t x+(1-t) y) \leqslant t f(x)+(1-t) f(y)
$$

for all $x, y \in I$ and $t \in[0,1]$.

The concept of convex functions has been generalized in different ways by many authors over the years. In [4], the author introduced the concept of s-convex functions as follows.

Definition 1.1 ([4]). A function $f:[0, \infty) \rightarrow \mathbb{R}$ is said to be $s$-convex (in the second sense), for $s \in(0,1]$, if

$$
f(t x+(1-t) y) \leqslant t^{s} f(x)+(1-t)^{s} f(y),
$$

for all $x, y \in[0, \infty)$ with $t \in[0,1]$.

The concept of m-convex functions was also introduced in [23] as follows.

Definition 1.2 ([23]). A function $f:[0, d] \rightarrow \mathbb{R}$ is said to be $m$-convex, for $m \in(0,1]$, if

$$
f(t x+m(1-t) y) \leqslant t f(x)+m(1-t) f(y),
$$

for all $x, y \in[0, d]$ with $t \in[0,1]$.

Email address: skermausour@alasu.edu (Seth Kermausuor)

doi: $10.22436 /$ jnsa.012.08.02

Received: 2018-12-27 Revised: 2019-02-20 Accepted: 2019-02-28 
By combining the concepts of $s$-convex and $m$-convex functions we have the concept of $(s, m)$-convex functions defined as follows.

Definition $1.3([10])$. A function $f:[0, \infty) \rightarrow \mathbb{R}$ is said to be $(s, m)$-convex (in the second sense), for $(s, \mathrm{~m}) \in(0,1]^{2}$, if

$$
f(t x+m(1-t) y) \leqslant t^{s} f(x)+m(1-t)^{s} f(y),
$$

for all $x, y \in[0, \infty)$ with $t \in[0,1]$.

In [19], Polyak also gave the following extension of convex functions to strongly convex functions.

Definition 1.4 ([19]). Given an interval $I$ in $\mathbb{R}$, a function $f: I \rightarrow \mathbb{R}$ is said to be strongly convex with modulus $\mu \geqslant 0$, if

$$
f(t x+(1-t) y) \leqslant t f(x)+(1-t) f(y)-\mu t(1-t)(y-x)^{2},
$$

for all $x, y \in I$ and $t \in[0,1]$.

Recently, Bracamonte et al. [3] introduced the concept of functions that are strongly ( $\mathrm{s}, \mathrm{m})$-convex in the second sense as follows.

Definition 1.5 ([3]). A function $f:[0, \infty) \rightarrow \mathbb{R}$ is said to be strongly $(s, m)$-convex function with modulus $\mu \geqslant 0$ (in the second sense), for $(s, m) \in(0,1]^{2}$, if

$$
f(t x+m(1-t) y) \leqslant t^{s} f(x)+m(1-t)^{s} f(y)-\mu t(1-t)(x-y)^{2},
$$

for all $x, y \in[0, \infty)$ and $t \in[0,1]$.

Over the years, many authors have studied and introduced several integral inequalities related to these classes of convex functions. For more information and related results, we refer the interested reader to the papers $[3,4,10,19,23]$.

In what follows, we review some fractional integral operators that are of importance to our current work.

Definition 1.6 ([18]). The left- and right-sided Riemann-Liouville fractional integrals of order $\alpha>0$ of $f$ are defined by

$$
J_{a^{+}}^{\alpha} f(x):=\frac{1}{\Gamma(\alpha)} \int_{a}^{x}(x-t)^{\alpha-1} f(t) d t
$$

and

$$
\mathrm{J}_{b^{-}}^{\alpha} f(x):=\frac{1}{\Gamma(\alpha)} \int_{x}^{b}(t-x)^{\alpha-1} f(t) d t,
$$

with $\mathrm{a}<\mathrm{x}<\mathrm{b}$ and $\Gamma(\cdot)$ is the gamma function given by

$$
\Gamma(x):=\int_{0}^{\infty} t^{x-1} e^{-t} d t, \quad \operatorname{Re}(x)>0,
$$

with the property that $\Gamma(x+1)=x \Gamma(x)$.

Definition 1.7 ([20]). The left- and right-sided Hadamard fractional integrals of order $\alpha>0$ of $f$ are defined by

$$
H_{a^{+}}^{\alpha} f(x):=\frac{1}{\Gamma(\alpha)} \int_{a}^{x}\left(\ln \frac{x}{t}\right)^{\alpha-1} \frac{f(t)}{t} d t
$$

and

$$
H_{b^{-}}^{\alpha} f(x):=\frac{1}{\Gamma(\alpha)} \int_{x}^{b}\left(\ln \frac{t}{x}\right)^{\alpha-1} \frac{f(t)}{t} d t
$$


Definition 1.8. $X_{c}^{p}(a, b) \quad(c \in \mathbb{R}, 1 \leqslant p \leqslant \infty)$ denotes the space of all complex-valued Lebesgue measurable functions $f$ for which $\|f\|_{X_{c}^{p}}<\infty$, where the norm $\|\cdot\|_{X_{c}^{p}}$ is defined by

$$
\|f\|_{X_{c}^{p}}=\left(\int_{a}^{b}\left|t^{c} f(t)\right|^{p} \frac{d t}{t}\right)^{1 / p}, \quad(1 \leqslant p<\infty),
$$

and for $p=\infty$

$$
\|f\|_{X_{c}^{\infty}}=\operatorname{ess} \sup _{a \leqslant t \leqslant b}\left|t^{c} f(t)\right| .
$$

In 2011, Katugampola introduced a new fractional integral operator which generalizes the RiemannLiouville and Hadamard fractional integrals as follows [14].

Definition 1.9. Let $[a, b] \subset \mathbb{R}$ be a finite interval. Then, the left- and right-sided Katugampola fractional integrals of order $\alpha>0$ of $f \in X_{c}^{p}(a, b)$ are defined by

$$
{ }^{\rho} I_{a+}^{\alpha} f(x):=\frac{\rho^{1-\alpha}}{\Gamma(\alpha)} \int_{a}^{x} \frac{t^{\rho-1}}{\left(x^{\rho}-t^{\rho}\right)^{1-\alpha}} f(t) d t
$$

and

$$
{ }^{\rho} I_{b-}^{\alpha} f(x):=\frac{\rho^{1-\alpha}}{\Gamma(\alpha)} \int_{x}^{b} \frac{t^{\rho-1}}{\left(t^{\rho}-x^{\rho}\right)^{1-\alpha}} f(t) d t
$$

with $a<x<b$ and $\rho>0$, if the integrals exist.

Remark 1.10. It is shown in [14] that the Katugampola fractional integral operators are well-defined on $X_{c}^{p}(a, b)$.

Theorem 1.11 ([14]). Let $\alpha>0$ and $\rho>0$. Then for $x>a$

1. $\lim _{\rho \rightarrow 1}{ }^{\rho} I_{a+}^{\alpha} f(x)=J_{a+}^{\alpha} f(x)$;

2. $\lim _{\rho \rightarrow 0^{+}}{ }^{\rho} \mathrm{I}_{a+}^{\alpha} f(x)=H_{a+}^{\alpha} f(x)$.

Similar results also hold for right-sided operators.

For more information about the Katugampola fractional integrals and related results, we refer the interested reader to the papers $[5,11,12,14,15,22]$.

In 1938, Ostrowski [17] obtained the following inequality which is known in the literature as Ostrowski inequality.

Theorem 1.12. Let $f:[a, b] \rightarrow \mathbb{R}$ be continuous on $[a, b]$ and differentiable in $(a, b)$ and its derivative $\mathrm{f}^{\prime}$ : $(\mathrm{a}, \mathrm{b}) \rightarrow \mathbb{R}$ is bounded in $(\mathrm{a}, \mathrm{b})$. If $M:=\sup _{\mathrm{t} \in(\mathrm{a}, \mathrm{b})}\left|\mathrm{f}^{\prime}(\mathrm{t})\right|<\infty$, then we have

$$
\left|f(x)-\frac{1}{b-a} \int_{a}^{b} f(t) d t\right| \leqslant\left(\frac{1}{4}+\frac{\left(x-\frac{a+b}{2}\right)^{2}}{(b-a)^{2}}\right)(b-a) M,
$$

for all $x \in[a, b]$. The inequality is sharp in the sense that the constant $\frac{1}{4}$ cannot be replaced by a smaller one.

Many authors have studied and generalized the Ostrowski inequality in several different ways. For more information about the Ostrowski inequality and its associates, we refer the interested reader to the papers $[1,2,6-9,11-13,21]$. The authors in $[1,11-13,21]$ provided some Ostrowski-type inequalities for some classes of convex functions.

Motivated by the results in the papers above and the current studies, the main goal of this paper is to provide some Ostrowski-type integral inequalities for functions whose second derivates belongs to the Lebesgue $\mathrm{L}_{\mathrm{q}}$ spaces and also for functions whose second derivatives at some powers are strongly $(s, \mathrm{~m})$-convex (in the second sense) via the Katugampola fractional integrals. 


\section{Main results}

To prove our main results, we need the following important lemma which is quite interesting in itself.

Lemma 2.1. Let $\alpha, \rho>0$, and $f:\left[a^{\rho}, b^{\rho}\right] \rightarrow \mathbb{R}$ be a twice differentiable function on $\left(a^{\rho}, b^{\rho}\right)$ with $0 \leqslant a<b$ such that $\mathrm{f}^{\prime \prime} \in \mathrm{L}_{1}\left(\left[\mathrm{a}^{\rho}, \mathrm{b}^{\rho}\right]\right)$. Then for all $\mathrm{x} \in[\mathrm{a}, \mathrm{b}]$, the following equality holds

$$
\begin{aligned}
& \frac{\rho^{\alpha} \Gamma(\alpha+1)}{b^{\rho}-a^{\rho}}\left[\frac{b^{\rho}-x^{\rho}}{\left(x^{\rho}-a^{\rho}\right)^{\alpha}} I_{x^{-}}^{\alpha} f\left(a^{\rho}\right)+\frac{x^{\rho}-a^{\rho}}{\left(b^{\rho}-x^{\rho}\right)^{\alpha}} I_{x^{+}}^{\alpha} f\left(b^{\rho}\right)\right]-f\left(x^{\rho}\right) \\
& =\frac{\left(x^{\rho}-a^{\rho}\right)\left(b^{\rho}-x^{\rho}\right)}{(\alpha+1)\left(b^{\rho}-a^{\rho}\right)}\left[\rho\left(x^{\rho}-a^{\rho}\right) \int_{0}^{1} t^{\alpha \rho+2 \rho-1} f^{\prime \prime}\left(t^{\rho} x^{\rho}+\left(1-t^{\rho}\right) a^{\rho}\right) d t\right. \\
& \left.\quad+\rho\left(b^{\rho}-x^{\rho}\right) \int_{0}^{1} t^{\alpha \rho+2 \rho-1} f^{\prime \prime}\left(t^{\rho} x^{\rho}+\left(1-t^{\rho}\right) b^{\rho}\right) d t\right] .
\end{aligned}
$$

Proof. The proof follows directly by using integration parts, change of variables and the definition of the Katugampola fractional integrals. First, we observe that

$$
\begin{aligned}
& \int_{0}^{1} t^{\alpha \rho+2 \rho-1} f^{\prime \prime}\left(t^{\rho} \chi^{\rho}+\left(1-t^{\rho}\right) a^{\rho}\right) d t \\
& =\int_{0}^{1} t^{\alpha \rho+\rho}\left[t^{\rho-1} f^{\prime \prime}\left(t^{\rho} \chi^{\rho}+\left(1-t^{\rho}\right) a^{\rho}\right)\right] d t \\
& =\left.\frac{1}{\rho\left(x^{\rho}-a^{\rho}\right)} t^{\alpha \rho+\rho} f^{\prime}\left(t^{\rho} \chi^{\rho}+\left(1-t^{\rho}\right) a^{\rho}\right)\right|_{0} ^{1} \\
& -\frac{\rho(\alpha+1)}{\rho\left(x^{\rho}-a^{\rho}\right)} \int_{0}^{1} t^{\alpha \rho+\rho-1} f^{\prime}\left(t^{\rho} \chi^{\rho}+\left(1-t^{\rho}\right) a^{\rho}\right) d t \\
& =\frac{1}{\rho\left(x^{\rho}-a^{\rho}\right)} f^{\prime}\left(x^{\rho}\right) \\
& -\frac{\alpha+1}{x^{\rho}-a^{\rho}} \int_{0}^{1} t^{\alpha \rho}\left[t^{\rho-1} f^{\prime}\left(t^{\rho} \chi^{\rho}+\left(1-t^{\rho}\right) a^{\rho}\right)\right] d t \\
& =\frac{1}{\rho\left(x^{\rho}-a^{\rho}\right)} f^{\prime}\left(x^{\rho}\right) \\
& -\frac{\alpha+1}{x^{\rho}-a^{\rho}}\left[\left.\frac{1}{\rho\left(x^{\rho}-a^{\rho}\right)} t^{\alpha \rho} f\left(t^{\rho} x^{\rho}+\left(1-t^{\rho}\right) a^{\rho}\right)\right|_{0} ^{1}\right. \\
& \left.-\frac{\rho \alpha}{\rho\left(x^{\rho}-a^{\rho}\right)} \int_{0}^{1} t^{\alpha \rho-1} f\left(t^{\rho} x^{\rho}+\left(1-t^{\rho}\right) a^{\rho}\right) d t\right] \\
& =\frac{1}{\rho\left(x^{\rho}-a^{\rho}\right)} f^{\prime}\left(x^{\rho}\right)-\frac{\alpha+1}{\rho\left(x^{\rho}-a^{\rho}\right)^{2}} f\left(x^{\rho}\right) \\
& +\frac{(\alpha+1) \alpha}{\left(x^{\rho}-a^{\rho}\right)^{2}} \int_{0}^{1} t^{\alpha \rho-1} f\left(t^{\rho} x^{\rho}+\left(1-t^{\rho}\right) a^{\rho}\right) d t \\
& =\frac{1}{\rho\left(x^{\rho}-a^{\rho}\right)} f^{\prime}\left(x^{\rho}\right)-\frac{\alpha+1}{\rho\left(x^{\rho}-a^{\rho}\right)^{2}} f\left(x^{\rho}\right) \\
& +\frac{(\alpha+1) \alpha}{\left(x^{\rho}-a^{\rho}\right)^{2}} \int_{0}^{1} t^{\rho(\alpha-1)} t^{\rho-1} f\left(t^{\rho} x^{\rho}+\left(1-t^{\rho}\right) a^{\rho}\right) d t \\
& =\frac{1}{\rho\left(x^{\rho}-a^{\rho}\right)} f^{\prime}\left(x^{\rho}\right)-\frac{\alpha+1}{\rho\left(x^{\rho}-a^{\rho}\right)^{2}} f\left(x^{\rho}\right) \\
& +\frac{(\alpha+1) \alpha}{\left(x^{\rho}-a^{\rho}\right)^{2}} \int_{a}^{x}\left(\frac{u^{\rho}-a^{\rho}}{x^{\rho}-a^{\rho}}\right)^{\alpha-1} f\left(u^{\rho}\right) \frac{u^{\rho-1}}{x^{\rho}-a^{\rho}} d u
\end{aligned}
$$




$$
\begin{aligned}
= & \frac{1}{\rho\left(x^{\rho}-a^{\rho}\right)} f^{\prime}\left(x^{\rho}\right)-\frac{\alpha+1}{\rho\left(x^{\rho}-a^{\rho}\right)^{2}} f\left(x^{\rho}\right) \\
& +\frac{(\alpha+1) \alpha}{\left(x^{\rho}-a^{\rho}\right)^{\alpha+2}} \int_{a}^{x}\left(u^{\rho}-a^{\rho}\right)^{\alpha-1} u^{\rho-1} f\left(u^{\rho}\right) d u \\
= & \frac{1}{\rho\left(x^{\rho}-a^{\rho}\right)} f^{\prime}\left(x^{\rho}\right)-\frac{\alpha+1}{\rho\left(x^{\rho}-a^{\rho}\right)^{2}} f\left(x^{\rho}\right) \\
& +\frac{(\alpha+1) \alpha}{\left(x^{\rho}-a^{\rho}\right)^{\alpha+2}} \frac{\Gamma(\alpha)}{\rho^{1-\alpha}} I_{x^{-}}^{\alpha} f\left(a^{\rho}\right) .
\end{aligned}
$$

Thus,

$$
\begin{aligned}
\int_{0}^{1} t^{\alpha \rho+2 \rho-1} f^{\prime \prime}\left(t^{\rho} x^{\rho}+\left(1-t^{\rho}\right) a^{\rho}\right) d t= & \frac{1}{\rho\left(x^{\rho}-a^{\rho}\right)} f^{\prime}\left(x^{\rho}\right) \\
& -\frac{\alpha+1}{\rho\left(x^{\rho}-a^{\rho}\right)^{2}} f\left(x^{\rho}\right)+\frac{\rho^{\alpha-1} \Gamma(\alpha+2)}{\left(x^{\rho}-a^{\rho}\right)^{\alpha+2}} I_{\chi^{-}}^{\alpha} f\left(a^{\rho}\right) .
\end{aligned}
$$

Similarly, we have

$$
\begin{aligned}
& \int_{0}^{1} t^{\alpha \rho+2 \rho-1} f^{\prime \prime}\left(t^{\rho} \chi^{\rho}+\left(1-t^{\rho}\right) b^{\rho}\right) d t \\
& =\int_{0}^{1} t^{\alpha \rho+\rho}\left[t^{\rho-1} f^{\prime \prime}\left(t^{\rho} \chi^{\rho}+\left(1-t^{\rho}\right) b^{\rho}\right)\right] d t \\
& =\left.\frac{1}{\rho\left(x^{\rho}-b^{\rho}\right)} t^{\alpha \rho+\rho} f^{\prime}\left(t^{\rho} \chi^{\rho}+\left(1-t^{\rho}\right) b^{\rho}\right)\right|_{0} ^{1} \\
& -\frac{\rho(\alpha+1)}{\rho\left(x^{\rho}-b^{\rho}\right)} \int_{0}^{1} t^{\alpha \rho+\rho-1} f^{\prime}\left(t^{\rho} x^{\rho}+\left(1-t^{\rho}\right) b^{\rho}\right) d t \\
& =\frac{1}{\rho\left(x^{\rho}-b^{\rho}\right)} f^{\prime}\left(x^{\rho}\right) \\
& -\frac{\alpha+1}{x^{\rho}-b^{\rho}} \int_{0}^{1} t^{\alpha \rho}\left[t^{\rho-1} f^{\prime}\left(t^{\rho} \chi^{\rho}+\left(1-t^{\rho}\right) b^{\rho}\right)\right] d t \\
& =\frac{1}{\rho\left(x^{\rho}-b^{\rho}\right)} f^{\prime}\left(x^{\rho}\right) \\
& -\frac{\alpha+1}{x^{\rho}-b^{\rho}}\left[\left.\frac{1}{\rho\left(x^{\rho}-b^{\rho}\right)} t^{\alpha \rho} f\left(t^{\rho} x^{\rho}+\left(1-t^{\rho}\right) b^{\rho}\right)\right|_{0} ^{1}\right. \\
& \left.-\frac{\rho \alpha}{\rho\left(x^{\rho}-b^{\rho}\right)} \int_{0}^{1} t^{\alpha \rho-1} f\left(t^{\rho} x^{\rho}+\left(1-t^{\rho}\right) b^{\rho}\right) d t\right] \\
& =\frac{1}{\rho\left(x^{\rho}-b^{\rho}\right)} f^{\prime}\left(x^{\rho}\right)-\frac{\alpha+1}{\rho\left(x^{\rho}-b^{\rho}\right)^{2}} f\left(x^{\rho}\right) \\
& +\frac{(\alpha+1) \alpha}{\left(x^{\rho}-b^{\rho}\right)^{2}} \int_{0}^{1} t^{\alpha \rho-1} f\left(t^{\rho} x^{\rho}+\left(1-t^{\rho}\right) b^{\rho}\right) d t \\
& =\frac{1}{\rho\left(x^{\rho}-b^{\rho}\right)} f^{\prime}\left(x^{\rho}\right)-\frac{\alpha+1}{\rho\left(x^{\rho}-b^{\rho}\right)^{2}} f\left(x^{\rho}\right) \\
& +\frac{(\alpha+1) \alpha}{\left(x^{\rho}-b^{\rho}\right)^{2}} \int_{0}^{1} t^{\rho(\alpha-1)} t^{\rho-1} f\left(t^{\rho} x^{\rho}+\left(1-t^{\rho}\right) b^{\rho}\right) d t \\
& =\frac{1}{\rho\left(x^{\rho}-b^{\rho}\right)} f^{\prime}\left(x^{\rho}\right)-\frac{\alpha+1}{\rho\left(x^{\rho}-b^{\rho}\right)^{2}} f\left(x^{\rho}\right) \\
& +\frac{(\alpha+1) \alpha}{\left(x^{\rho}-b^{\rho}\right)^{2}} \int_{b}^{x}\left(\frac{u^{\rho}-b^{\rho}}{x^{\rho}-b^{\rho}}\right)^{\alpha-1} f\left(u^{\rho}\right) \frac{u^{\rho-1}}{x^{\rho}-b^{\rho}} d u
\end{aligned}
$$




$$
\begin{aligned}
= & \frac{1}{\rho\left(x^{\rho}-b^{\rho}\right)} f^{\prime}\left(x^{\rho}\right)-\frac{\alpha+1}{\rho\left(x^{\rho}-b^{\rho}\right)^{2}} f\left(x^{\rho}\right) \\
& +\frac{(\alpha+1) \alpha}{\left(x^{\rho}-b^{\rho}\right)^{2}} \int_{x}^{b}\left(\frac{b^{\rho}-u^{\rho}}{b^{\rho}-x^{\rho}}\right)^{\alpha-1} f\left(u^{\rho}\right) \frac{u^{\rho-1}}{b^{\rho}-x^{\rho}} d u \\
= & \frac{1}{\rho\left(x^{\rho}-b^{\rho}\right)} f^{\prime}\left(x^{\rho}\right)-\frac{\alpha+1}{\rho\left(x^{\rho}-b^{\rho}\right)^{2}} f\left(x^{\rho}\right) \\
& +\frac{(\alpha+1) \alpha}{\left(b^{\rho}-x^{\rho}\right)^{\alpha+2}} \int_{x}^{b}\left(b^{\rho}-u^{\rho}\right)^{\alpha-1} u^{\rho-1} f\left(u^{\rho}\right) d u \\
= & \frac{1}{\rho\left(x^{\rho}-b^{\rho}\right)} f^{\prime}\left(x^{\rho}\right)-\frac{\alpha+1}{\rho\left(x^{\rho}-b^{\rho}\right)^{2}} f\left(x^{\rho}\right) \\
& +\frac{(\alpha+1) \alpha}{\left(b^{\rho}-x^{\rho}\right)^{\alpha+2}} \frac{\Gamma(\alpha)}{\rho^{1-\alpha}} I_{x^{+}}^{\alpha} f\left(b^{\rho}\right) .
\end{aligned}
$$

Thus,

$$
\begin{aligned}
\int_{0}^{1} t^{\alpha \rho+2 \rho-1} f^{\prime \prime}\left(t^{\rho} \chi^{\rho}+\left(1-t^{\rho}\right) b^{\rho}\right) d t= & -\frac{1}{\rho\left(b^{\rho}-x^{\rho}\right)} f^{\prime}\left(x^{\rho}\right)-\frac{\alpha+1}{\rho\left(b^{\rho}-x^{\rho}\right)^{2}} f\left(x^{\rho}\right) \\
& +\frac{\rho^{\alpha-1} \Gamma(\alpha+2)}{\left(b^{\rho}-x^{\rho}\right)^{\alpha+2}}{ }^{\alpha} \chi^{\alpha} f\left(b^{\rho}\right) .
\end{aligned}
$$

Now, we multiply (2.2) by $\rho\left(x^{\rho}-a^{\rho}\right)$ and (2.3) by $\rho\left(b^{\rho}-x^{\rho}\right)$, then add the results to get

$$
\begin{aligned}
& \rho\left(x^{\rho}-a^{\rho}\right) \int_{0}^{1} t^{\alpha \rho+2 \rho-1} f^{\prime \prime}\left(t^{\rho} x^{\rho}+\left(1-t^{\rho}\right) a^{\rho}\right) d t \\
& +\rho\left(b^{\rho}-x^{\rho}\right) \int_{0}^{1} t^{\alpha \rho+2 \rho-1} f^{\prime \prime}\left(t^{\rho} x^{\rho}+\left(1-t^{\rho}\right) b^{\rho}\right) d t \\
& =\frac{\rho^{\alpha} \Gamma(\alpha+2)}{\left(x^{\rho}-a^{\rho}\right)^{\alpha+1}}{ }^{\rho} \chi_{\chi^{-}}^{\alpha} f\left(a^{\rho}\right) \\
& +\frac{\rho^{\alpha} \Gamma(\alpha+2)}{\left(b^{\rho}-x^{\rho}\right)^{\alpha+1}}{ }^{\rho} x_{x^{+}}^{\alpha} f\left(b^{\rho}\right) \\
& -\frac{\alpha+1}{x^{\rho}-a^{\rho}} f\left(x^{\rho}\right)-\frac{\alpha+1}{b^{\rho}-x^{\rho}} f\left(x^{\rho}\right) \\
& =\frac{\rho^{\alpha} \Gamma(\alpha+2)}{\left(x^{\rho}-a^{\rho}\right)^{\alpha+1}}{ }^{\alpha} \mathrm{I}^{-} f\left(a^{\rho}\right) \\
& +\frac{\rho^{\alpha} \Gamma(\alpha+2)}{\left(b^{\rho}-x^{\rho}\right)^{\alpha+1}}{ }^{\rho} x_{x^{+}}^{\alpha} f\left(b^{\rho}\right) \\
& -\frac{(\alpha+1)\left(b^{\rho}-a^{\rho}\right)}{\left(x^{\rho}-a^{\rho}\right)\left(b^{\rho}-x^{\rho}\right)} f\left(x^{\rho}\right) \text {. }
\end{aligned}
$$

Multiplying (2.4) by $\frac{\left(x^{\rho}-a^{\rho}\right)\left(b^{\rho}-x^{\rho}\right)}{(\alpha+1)\left(b^{\rho}-a^{\rho}\right)}$ gives the desired identity in (2.1).

Remark 2.2. If $\rho=1$, then Lemma 2.1 reduces to [16, Lemma 2.1].

Theorem 2.3. Under the conditions of Lemma 2.1, suppose that $\mathrm{f}^{\prime \prime}$ is bounded, i.e., $\left\|f^{\prime \prime}\right\|_{\infty}=\sup _{\mathfrak{t} \in\left[\mathbf{a}^{\rho}, \mathrm{b}^{\rho}\right]}\left|\mathrm{f}^{\prime \prime}(\mathrm{t})\right|<\infty$. Then for any $x \in[a, b]$, the following inequality holds:

$$
\left|\frac{\rho^{\alpha} \Gamma(\alpha+1)}{b^{\rho}-a^{\rho}}\left[\frac{b^{\rho}-x^{\rho}}{\left(x^{\rho}-a^{\rho}\right)^{\alpha}}{ }^{\rho} x^{\alpha} f\left(a^{\rho}\right)+\frac{x^{\rho}-a^{\rho}}{\left(b^{\rho}-x^{\rho}\right)^{\alpha}}{ }^{\rho} I_{x^{+}}^{\alpha} f\left(b^{\rho}\right)\right]-f\left(x^{\rho}\right)\right| \leqslant \frac{\left(x^{\rho}-a^{\rho}\right)\left(b^{\rho}-x^{\rho}\right)}{(\alpha+1)(\alpha+2)}\left\|f^{\prime \prime}\right\|_{\infty} .
$$


Proof. Using Lemma 2.1 and the properties of the absolute value, we have

$$
\begin{aligned}
& \left|\frac{\rho^{\alpha} \Gamma(\alpha+1)}{b^{\rho}-a^{\rho}}\left[\frac{b^{\rho}-x^{\rho}}{\left(x^{\rho}-a^{\rho}\right)^{\alpha}} I_{x^{-}}^{\alpha} f\left(a^{\rho}\right)+\frac{x^{\rho}-a^{\rho}}{\left(b^{\rho}-x^{\rho}\right)^{\alpha}} I_{x^{+}}^{\alpha} f\left(b^{\rho}\right)\right]-f\left(x^{\rho}\right)\right| \\
& \leqslant \\
& \quad \frac{\left(x^{\rho}-a^{\rho}\right)\left(b^{\rho}-x^{\rho}\right)}{(\alpha+1)\left(b^{\rho}-a^{\rho}\right)}\left[\rho\left(x^{\rho}-a^{\rho}\right) \int_{0}^{1} t^{\alpha \rho+2 \rho-1}\left|f^{\prime \prime}\left(t^{\rho} x^{\rho}+\left(1-t^{\rho}\right) a^{\rho}\right)\right| d t\right. \\
& \left.\quad+\rho\left(b^{\rho}-x^{\rho}\right) \int_{0}^{1} t^{\alpha \rho+2 \rho-1}\left|f^{\prime \prime}\left(t^{\rho} x^{\rho}+\left(1-t^{\rho}\right) b^{\rho}\right)\right| d t\right] \\
& \leqslant \\
& \quad \frac{\left(x^{\rho}-a^{\rho}\right)\left(b^{\rho}-x^{\rho}\right)}{(\alpha+1)\left(b^{\rho}-a^{\rho}\right)}\left[\left\|f^{\prime \prime}\right\|_{\infty} \rho\left(b^{\rho}-a^{\rho}\right) \int_{0}^{1} t^{\alpha \rho+2 \rho-1} d t\right] \\
& =\frac{\left(x^{\rho}-a^{\rho}\right)\left(b^{\rho}-x^{\rho}\right)}{(\alpha+1)\left(b^{\rho}-a^{\rho}\right)}\left[\frac{\rho\left(b^{\rho}-a^{\rho}\right)}{\rho(\alpha+2)}\left\|f^{\prime \prime}\right\|_{\infty}\right] \\
& =\frac{\left(x^{\rho}-a^{\rho}\right)\left(b^{\rho}-x^{\rho}\right)}{(\alpha+1)(\alpha+2)}\left\|f^{\prime \prime}\right\|_{\infty} .
\end{aligned}
$$

This completes the proof.

Theorem 2.4. Under the conditions of Lemma 2.1, suppose that $f^{\prime \prime} \in \mathrm{L}_{1}\left(\left[\mathrm{a}^{\rho}, \mathrm{b}^{\rho}\right]\right)$. Then for any $\mathrm{x} \in[\mathrm{a}, \mathrm{b}]$, the following inequality holds:

$$
\left|\frac{\rho^{\alpha} \Gamma(\alpha+1)}{b^{\rho}-a^{\rho}}\left[\frac{b^{\rho}-x^{\rho}}{\left(x^{\rho}-a^{\rho}\right)^{\alpha}} I_{x^{-}}^{\alpha} f\left(a^{\rho}\right)+\frac{x^{\rho}-a^{\rho}}{\left(b^{\rho}-x^{\rho}\right)^{\alpha}} I_{x^{+}}^{\alpha} f\left(b^{\rho}\right)\right]-f\left(x^{\rho}\right)\right| \leqslant \frac{\left(x^{\rho}-a^{\rho}\right)\left(b^{\rho}-x^{\rho}\right)}{(\alpha+1)\left(b^{\rho}-a^{\rho}\right)}\left\|f^{\prime \prime}\right\|_{1},
$$

where $\left\|f^{\prime \prime}\right\|_{1}=\int_{a^{\rho}}^{b^{\rho}}\left|f^{\prime \prime}(u)\right| d u<\infty$.

Proof. Using Lemma 2.1 and the properties of the absolute value, we have

$$
\begin{aligned}
&\left|\frac{\rho^{\alpha} \Gamma(\alpha+1)}{b^{\rho}-a^{\rho}}\left[\frac{b^{\rho}-x^{\rho}}{\left(x^{\rho}-a^{\rho}\right)^{\rho}} I_{x^{-}}^{\alpha} f\left(a^{\rho}\right)+\frac{x^{\rho}-a^{\rho}}{\left(b^{\rho}-x^{\rho}\right)^{\alpha}} I_{x^{+}}^{\alpha} f\left(b^{\rho}\right)\right]-f\left(x^{\rho}\right)\right| \\
& \leqslant \frac{\left(x^{\rho}-a^{\rho}\right)\left(b^{\rho}-x^{\rho}\right)}{(\alpha+1)\left(b^{\rho}-a^{\rho}\right)}\left[\rho\left(x^{\rho}-a^{\rho}\right) \int_{0}^{1} t^{\alpha \rho+\rho} t^{\rho-1}\left|f^{\prime \prime}\left(t^{\rho} x^{\rho}+\left(1-t^{\rho}\right) a^{\rho}\right)\right| d t\right. \\
&\left.+\rho\left(b^{\rho}-x^{\rho}\right) \int_{0}^{1} t^{\alpha \rho+\rho} t^{\rho-1}\left|f^{\prime \prime}\left(t^{\rho} x^{\rho}+\left(1-t^{\rho}\right) b^{\rho}\right)\right| d t\right] \\
& \leqslant \frac{\left(x^{\rho}-a^{\rho}\right)\left(b^{\rho}-x^{\rho}\right)}{(\alpha+1)\left(b^{\rho}-a^{\rho}\right)}\left[\rho\left(x^{\rho}-a^{\rho}\right) \int_{0}^{1} t^{\rho-1}\left|f^{\prime \prime}\left(t^{\rho} x^{\rho}+\left(1-t^{\rho}\right) a^{\rho}\right)\right| d t\right. \\
&\left.+\rho\left(b^{\rho}-x^{\rho}\right) \int_{0}^{1} t^{\rho-1}\left|f^{\prime \prime}\left(t^{\rho} x^{\rho}+\left(1-t^{\rho}\right) b^{\rho}\right)\right| d t\right] \\
&= \frac{\left(x^{\rho}-a^{\rho}\right)\left(b^{\rho}-x^{\rho}\right)}{(\alpha+1)\left(b^{\rho}-a^{\rho}\right)}\left[\int_{a^{\rho}}^{x^{\rho}}\left|f^{\prime \prime}(u)\right| d u+\int_{x^{\rho}}^{b^{\rho}}\left|f^{\prime \prime}(u)\right| d u\right] \\
&= \frac{\left(x^{\rho}-a^{\rho}\right)\left(b^{\rho}-x^{\rho}\right)}{(\alpha+1)\left(b^{\rho}-a^{\rho}\right)} \int_{a^{\rho}}^{b^{\rho}}\left|f^{\prime \prime}(u)\right| d u=\frac{\left(x^{\rho}-a^{\rho}\right)\left(b^{\rho}-x^{\rho}\right)}{(\alpha+1)\left(b^{\rho}-a^{\rho}\right)}\left\|f^{\prime \prime}\right\|_{1} .
\end{aligned}
$$

This completes the proof.

Theorem 2.5. Under the conditions of Lemma 2.1, suppose that $\mathrm{f}^{\prime \prime} \in \mathrm{L}_{\mathrm{q}}\left(\left[\mathrm{a}^{\rho}, \mathrm{b}^{\rho}\right]\right)$ with $1<\mathrm{q}<\infty$. Then for any $x \in[a, b]$, the following inequality holds:

$$
\left|\frac{\rho^{\alpha} \Gamma(\alpha+1)}{b^{\rho}-a^{\rho}}\left[\frac{b^{\rho}-x^{\rho}}{\left(x^{\rho}-a^{\rho}\right)^{\alpha}}{ }^{\rho} I_{x^{-}}^{\alpha} f\left(a^{\rho}\right)+\frac{x^{\rho}-a^{\rho}}{\left(b^{\rho}-x^{\rho}\right)^{\alpha}}{ }^{\rho} I_{\chi^{+}}^{\alpha} f\left(b^{\rho}\right)\right]-f\left(x^{\rho}\right)\right|
$$




$$
\leqslant \frac{2\left(x^{\rho}-a^{\rho}\right)\left(b^{\rho}-x^{\rho}\right)}{(\alpha+1)\left(b^{\rho}-a^{\rho}\right)^{\frac{1}{q}}}\left(\frac{1}{r(\alpha+1)}\right)^{\frac{1}{r}}\left\|f^{\prime \prime}\right\|_{q},
$$

where $\left\|f^{\prime \prime}\right\|_{\mathrm{q}}=\left(\int_{a^{\rho}}^{\mathrm{b}^{\rho}}\left|f^{\prime \prime}(\mathrm{u})\right|^{\mathrm{q}} \mathrm{du}\right)^{\frac{1}{q}}<\infty$ and $\frac{1}{\mathrm{r}}+\frac{1}{\mathrm{q}}=1$.

Proof. Using Lemma 2.1, the fact that $t^{\rho-1}=t^{\frac{\rho-1}{r}} t^{\frac{\rho-1}{q}}$ and the Hölder's inequality, we have

$$
\begin{aligned}
& \left|\frac{\rho^{\alpha} \Gamma(\alpha+1)}{b^{\rho}-a^{\rho}}\left[\frac{b^{\rho}-x^{\rho}}{\left(x^{\rho}-a^{\rho}\right)^{\alpha}}{ }^{\rho} I_{x^{-}}^{\alpha} f\left(a^{\rho}\right)+\frac{x^{\rho}-a^{\rho}}{\left(b^{\rho}-x^{\rho}\right)^{\alpha}} I_{x^{+}}^{\alpha} f\left(b^{\rho}\right)\right]-f\left(x^{\rho}\right)\right| \\
& \leqslant \frac{\left(x^{\rho}-a^{\rho}\right)\left(b^{\rho}-x^{\rho}\right)}{(\alpha+1)\left(b^{\rho}-a^{\rho}\right)}\left[\rho\left(x^{\rho}-a^{\rho}\right) \int_{0}^{1} t^{\alpha \rho+\rho} t^{\rho-1}\left|f^{\prime \prime}\left(t^{\rho} x^{\rho}+\left(1-t^{\rho}\right) a^{\rho}\right)\right| d t\right. \\
& \left.+\rho\left(b^{\rho}-x^{\rho}\right) \int_{0}^{1} t^{\alpha \rho+\rho} t^{\rho-1}\left|f^{\prime \prime}\left(t^{\rho} x^{\rho}+\left(1-t^{\rho}\right) b^{\rho}\right)\right| d t\right] \\
& \leqslant \frac{\rho\left(x^{\rho}-a^{\rho}\right)\left(b^{\rho}-x^{\rho}\right)}{(\alpha+1)\left(b^{\rho}-a^{\rho}\right)}\left(\int_{0}^{1} t^{\rho r(\alpha+1)} t^{\rho-1} d t\right)^{\frac{1}{r}} \\
& \times\left[\left(x^{\rho}-a^{\rho}\right)\left(\int_{0}^{1} t^{\rho-1}\left|f^{\prime \prime}\left(t^{\rho} x^{\rho}+\left(1-t^{\rho}\right) a^{\rho}\right)\right|^{q} d t\right)^{\frac{1}{q}}\right. \\
& \left.+\left(b^{\rho}-x^{\rho}\right)\left(\int_{0}^{1} t^{\rho-1}\left|f^{\prime \prime}\left(t^{\rho} x^{\rho}+\left(1-t^{\rho}\right) b^{\rho}\right)\right|^{q} d t\right)^{\frac{1}{q}}\right] \\
& =\frac{\rho\left(x^{\rho}-a^{\rho}\right)\left(b^{\rho}-x^{\rho}\right)}{(\alpha+1)\left(b^{\rho}-a^{\rho}\right)}\left(\frac{1}{\rho r(\alpha+1)}\right)^{\frac{1}{r}} \\
& \times\left[\left(x^{\rho}-a^{\rho}\right)\left(\frac{1}{\rho\left(x^{\rho}-a^{\rho}\right)} \int_{a^{\rho}}^{x^{\rho}}\left|f^{\prime \prime}(u)\right|^{q} d u\right)^{\frac{1}{q}}\right. \\
& \left.+\left(b^{\rho}-x^{\rho}\right)\left(\frac{1}{\rho\left(b^{\rho}-x^{\rho}\right)} \int_{x^{\rho}}^{x^{\rho}}\left|f^{\prime \prime}(u)\right|^{q} d u\right)^{\frac{1}{q}}\right] \\
& =\frac{\left(x^{\rho}-a^{\rho}\right)\left(b^{\rho}-x^{\rho}\right)}{(\alpha+1)\left(b^{\rho}-a^{\rho}\right)}\left(\frac{1}{r(\alpha+1)}\right)^{\frac{1}{r}} \\
& \times\left[\left(x^{\rho}-a^{\rho}\right)^{1-\frac{1}{q}}\left(\int_{a^{\rho}}^{x^{\rho}}\left|f^{\prime \prime}(u)\right|^{q} d u\right)^{\frac{1}{q}}\right. \\
& \left.+\left(b^{\rho}-x^{\rho}\right)^{1-\frac{1}{q}}\left(\int_{x^{\rho}}^{x^{\rho}}\left|f^{\prime \prime}(u)\right|^{q} d u\right)^{\frac{1}{q}}\right] \\
& \leqslant \frac{\left(x^{\rho}-a^{\rho}\right)\left(b^{\rho}-x^{\rho}\right)}{(\alpha+1)\left(b^{\rho}-a^{\rho}\right)}\left(\frac{1}{r(\alpha+1)}\right)^{\frac{1}{r}}\left(2\left(b^{\rho}-a^{\rho}\right)^{1-\frac{1}{q}}\left\|f^{\prime \prime}\right\|_{q}\right) \text {. }
\end{aligned}
$$

This completes the proof.

Theorem 2.6. Let $\alpha, \rho>0$ and $\mathrm{f}:[0, \infty) \rightarrow \mathbb{R}$ be a twice differentiable function on $\left(\mathrm{a}^{\rho}, \mathrm{b}^{\rho}\right)$ such that $\mathrm{f}^{\prime \prime} \in$ $\mathrm{L}_{1}\left(\left[\mathrm{a}^{\rho}, \mathrm{b}^{\rho}\right]\right)$ where $0 \leqslant \mathrm{a}<\mathrm{b}$. If $\left|\mathrm{f}^{\prime \prime}\right|^{\mathrm{q}}$ is strongly $(\mathrm{s}, \mathrm{m})$-convex with modulus $\mu \geqslant 0$ (in the second sense) and 
$(s, m) \in(0,1]^{2}$, for $q \geqslant 1$, then for any $x \in[a, b]$, the following inequality holds:

$$
\begin{aligned}
\mid \frac{\rho^{\alpha} \Gamma(\alpha+1)}{b^{\rho}-a^{\rho}} & {\left[\frac{b^{\rho}-x^{\rho}}{\left(x^{\rho}-a^{\rho}\right)^{\alpha}} I_{x^{-}}^{\alpha} f\left(a^{\rho}\right)+\frac{x^{\rho}-a^{\rho}}{\left(b^{\rho}-x^{\rho}\right)^{\alpha}} I_{x^{+}}^{\alpha} f\left(b^{\rho}\right)\right]-f\left(x^{\rho}\right) \mid } \\
\leqslant & \frac{\left(x^{\rho}-a^{\rho}\right)\left(b^{\rho}-x^{\rho}\right)}{(\alpha+1)\left(b^{\rho}-a^{\rho}\right)}\left(\frac{1}{\alpha+2}\right)^{1-\frac{1}{q}} \\
& \times\left[( x ^ { \rho } - a ^ { \rho } ) \left(\frac{\left|f^{\prime \prime}\left(x^{\rho}\right)\right|^{q}}{\alpha+s+2}+m B(\alpha+2, s+1)\left|f^{\prime \prime}\left(\frac{a^{\rho}}{m}\right)\right|^{q}\right.\right. \\
& \left.-\frac{\mu}{(\alpha+3)(\alpha+4)}\left(\frac{a^{\rho}}{m}-x^{\rho}\right)^{2}\right)^{\frac{1}{q}} \\
& +\left(b^{\rho}-x^{\rho}\right)\left(\frac{\left|f^{\prime \prime}\left(x^{\rho}\right)\right|^{q}}{\alpha+s+2}+m B(\alpha+2, s+1)\left|f^{\prime \prime}\left(\frac{b^{\rho}}{m}\right)\right|^{q}\right. \\
& \left.\left.-\frac{\mu}{(\alpha+3)(\alpha+4)}\left(\frac{b^{\rho}}{m}-x^{\rho}\right)^{2}\right)^{\frac{1}{q}}\right],
\end{aligned}
$$

where $\mathrm{B}(\cdot, \cdot)$ denotes the beta function defined by

$$
\mathrm{B}(\mathrm{u}, v)=\int_{0}^{1} \mathrm{t}^{\mathrm{u}-1}(1-\mathrm{t})^{v-1} \mathrm{dt}, \quad \mathrm{u}, v>0
$$

Proof. Using Lemma 2.1, the power mean inequality and the strong $(s, m)$-convexity of $\left|f^{\prime \prime}\right| q$, we have

$$
\begin{aligned}
&\left|\frac{\rho^{\alpha} \Gamma(\alpha+1)}{b^{\rho}-a^{\rho}}\left[\frac{b^{\rho}-x^{\rho}}{\left(x^{\rho}-a^{\rho}\right)^{\rho}} I_{x^{-}}^{\alpha} f\left(a^{\rho}\right)+\frac{x^{\rho}-a^{\rho}}{\left(b^{\rho}-x^{\rho}\right)^{\rho}} I_{x^{+}}^{\alpha} f\left(b^{\rho}\right)\right]-f\left(x^{\rho}\right)\right| \\
& \leqslant \frac{\left(x^{\rho}-a^{\rho}\right)\left(b^{\rho}-x^{\rho}\right)}{(\alpha+1)\left(b^{\rho}-a^{\rho}\right)}\left[\rho\left(x^{\rho}-a^{\rho}\right) \int_{0}^{1} t^{\alpha \rho+2 \rho-1}\left|f^{\prime \prime}\left(t^{\rho} x^{\rho}+\left(1-t^{\rho}\right) a^{\rho}\right)\right| d t\right. \\
&\left.+\rho\left(b^{\rho}-x^{\rho}\right) \int_{0}^{1} t^{\alpha \rho+2 \rho-1}\left|f^{\prime \prime}\left(t^{\rho} x^{\rho}+\left(1-t^{\rho}\right) b^{\rho}\right)\right| d t\right] \\
& \leqslant \frac{\rho\left(x^{\rho}-a^{\rho}\right)\left(b^{\rho}-x^{\rho}\right)}{(\alpha+1)\left(b^{\rho}-a^{\rho}\right)}\left(\int_{0}^{1} t^{\alpha \rho+2 \rho-1} d t\right)^{1-\frac{1}{q}} \\
& \times\left[\left(x^{\rho}-a^{\rho}\right)\left(\int_{0}^{1} t^{\alpha \rho+2 \rho-1}\left|f^{\prime \prime}\left(t^{\rho} x^{\rho}+\left(1-t^{\rho}\right) a^{\rho}\right)\right|^{q} d t\right)^{\frac{1}{q}}\right. \\
&\left.+\left(b^{\rho}-x^{\rho}\right)\left(\int_{0}^{1} t^{\alpha \rho+2 \rho-1}\left|f^{\prime \prime}\left(t^{\rho} x^{\rho}+\left(1-t^{\rho}\right) b^{\rho}\right)\right| d t\right)^{\frac{1}{q}}\right] \\
& \leqslant \frac{\rho\left(x^{\rho}-a^{\rho}\right)\left(b^{\rho}-x^{\rho}\right)}{(\alpha+1)\left(b^{\rho}-a^{\rho}\right)}\left(\frac{1}{\rho(\alpha+2)}\right)^{1-\frac{1}{q}} \\
& \times\left[( x ^ { \rho } - a ^ { \rho } ) \left(\int _ { 0 } ^ { 1 } t ^ { \alpha \rho + 2 \rho - 1 } \left(t^{s \rho}\left|f^{\prime \prime}\left(x^{\rho}\right)\right|^{q}+m\left(1-t^{\rho}\right)^{s}\left|f^{\prime \prime}\left(\frac{a^{\rho}}{m}\right)\right|^{q}\right.\right.\right. \\
&\left.\left.-\mu t^{\rho}\left(1-t^{\rho}\right)\left(\frac{a^{\rho}}{m}-x^{\rho}\right)^{2}\right) d t\right)^{\frac{1}{q}} \\
&+\left(b^{\rho}-x^{\rho}\right)\left(\left.\int_{0}^{1} t^{\alpha \rho+2 \rho-1}\left(t^{s \rho}\left|f^{\prime \prime}\left(x^{\rho}\right)\right|^{q}+m\left(1-t^{\rho}\right)\left(\frac{b^{\rho}}{m}-x^{\rho}\right)^{2}\right) d t f^{\prime \prime}\left(\frac{b^{\rho}}{m}\right)\right|^{q}\right. \\
& {[1}
\end{aligned}
$$




$$
\begin{aligned}
= & \frac{\rho\left(x^{\rho}-a^{\rho}\right)\left(b^{\rho}-x^{\rho}\right)}{(\alpha+1)\left(b^{\rho}-a^{\rho}\right)}\left(\frac{1}{\rho(\alpha+2)}\right)^{1-\frac{1}{q}} \\
& \times\left[( x ^ { \rho } - a ^ { \rho } ) \left(\left|f^{\prime \prime}\left(x^{\rho}\right)\right|^{q} \int_{0}^{1} t^{\alpha \rho+s \rho+2 \rho-1} d t+m\left|f^{\prime \prime}\left(\frac{a^{\rho}}{m}\right)\right|^{q} \int_{0}^{1} t^{\alpha \rho+2 \rho-1}\left(1-t^{\rho}\right)^{s} d t\right.\right. \\
& \left.-\mu\left(\frac{a^{\rho}}{m}-x^{\rho}\right)^{2} \int_{0}^{1} t^{\alpha \rho+2 \rho-1} t^{\rho}\left(1-t^{\rho}\right) d t\right)^{\frac{1}{q}} \\
& +\left(b^{\rho}-x^{\rho}\right)\left(\left|f^{\prime \prime}\left(x^{\rho}\right)\right|^{q} \int_{0}^{1} t^{\alpha \rho+s \rho+2 \rho-1} d t+m\left|f^{\prime \prime}\left(\frac{b^{\rho}}{m}\right)\right|^{q} \int_{0}^{1} t^{\alpha \rho+2 \rho-1}\left(1-t^{\rho}\right)^{s} d t\right. \\
& \left.\left.-\mu\left(\frac{b^{\rho}}{m}-x^{\rho}\right)^{2} \int_{0}^{1} t^{\alpha \rho+2 \rho-1} t^{\rho}\left(1-t^{\rho}\right) d t\right)^{\frac{1}{q}}\right] .
\end{aligned}
$$

The desired inequality follows from the above by noting that

$$
\int_{0}^{1} t^{\alpha \rho+s \rho+2 \rho-1} d t=\frac{1}{\rho(\alpha+s+2)}, \quad \int_{0}^{1} t^{\alpha \rho+2 \rho-1} t^{\rho}\left(1-t^{\rho}\right) d t=\frac{1}{\rho(\alpha+3)(\alpha+4)},
$$

and

$$
\begin{aligned}
\int_{0}^{1} t^{\alpha \rho+2 \rho-1}\left(1-t^{\rho}\right)^{s} d t & =\int_{0}^{1} t^{\alpha \rho+\rho}\left(1-t^{\rho}\right)^{s} t^{\rho-1} d t \\
& =\frac{1}{\rho} \int_{0}^{1} u^{\alpha+1}(1-u)^{s} d u \\
& =\frac{1}{\rho} B(\alpha+2, s+1) .
\end{aligned}
$$

This completes the proof.

Corollary 2.7. Under the conditions of Theorem 2.6,

1. if $\left|f^{\prime \prime}\right|^{q}$ is s-convex, i.e., $\mathrm{m}=1$ and $\mu=0$, then we have the inequality

$$
\begin{aligned}
\mid \frac{\rho^{\alpha} \Gamma(\alpha+1)}{b^{\rho}-a^{\rho}} & {\left[\frac{b^{\rho}-x^{\rho}}{\left(x^{\rho}-a^{\rho}\right)^{\alpha}}{ }^{\rho}{x^{-}}^{\alpha} f\left(a^{\rho}\right)+\frac{x^{\rho}-a^{\rho}}{\left(b^{\rho}-x^{\rho}\right)^{\alpha}}{ }^{\rho} x_{x^{+}}^{\alpha} f\left(b^{\rho}\right)\right]-f\left(x^{\rho}\right) \mid } \\
\leqslant & \frac{\left(x^{\rho}-a^{\rho}\right)\left(b^{\rho}-x^{\rho}\right)}{(\alpha+1)\left(b^{\rho}-a^{\rho}\right)}\left(\frac{1}{\alpha+2}\right)^{1-\frac{1}{q}} \\
& \times\left[\left(x^{\rho}-a^{\rho}\right)\left(\frac{\left|f^{\prime \prime}\left(x^{\rho}\right)\right|^{q}}{\alpha+s+2}+B(\alpha+2, s+1)\left|f^{\prime \prime}\left(a^{\rho}\right)\right|^{q}\right)^{\frac{1}{q}}\right. \\
& \left.+\left(b^{\rho}-x^{\rho}\right)\left(\frac{\left|f^{\prime \prime}\left(x^{\rho}\right)\right|^{q}}{\alpha+s+2}+B(\alpha+2, s+1)\left|f^{\prime \prime}\left(b^{\rho}\right)\right|^{q}\right)^{\frac{1}{q}}\right] ;
\end{aligned}
$$

2. if $\left|f^{\prime \prime}\right|^{\mathrm{q}}$ is $\mathrm{m}$-convex, i.e., $s=1$ and $\mu=0$, then we have the inequality

$$
\begin{aligned}
&\left|\frac{\rho^{\alpha} \Gamma(\alpha+1)}{b^{\rho}-a^{\rho}}\left[\frac{b^{\rho}-x^{\rho}}{\left(x^{\rho}-a^{\rho}\right)^{\alpha}} I_{x^{-}}^{\alpha} f\left(a^{\rho}\right)+\frac{x^{\rho}-a^{\rho}}{\left(b^{\rho}-x^{\rho}\right)^{\alpha}} I_{x^{+}}^{\alpha} f\left(b^{\rho}\right)\right]-f\left(x^{\rho}\right)\right| \\
& \leqslant \frac{\left(x^{\rho}-a^{\rho}\right)\left(b^{\rho}-x^{\rho}\right)}{(\alpha+1)\left(b^{\rho}-a^{\rho}\right)}\left(\frac{1}{\alpha+2}\right)^{1-\frac{1}{q}} \\
& \times\left[\left(x^{\rho}-a^{\rho}\right)\left(\frac{\left|f^{\prime \prime}\left(x^{\rho}\right)\right|^{q}}{\alpha+3}+\frac{m}{(\alpha+2)(\alpha+3)}\left|f^{\prime \prime}\left(\frac{a^{\rho}}{m}\right)\right|^{q}\right)^{\frac{1}{q}}\right. \\
&\left.+\left(b^{\rho}-x^{\rho}\right)\left(\frac{\left|f^{\prime \prime}\left(x^{\rho}\right)\right|^{q}}{\alpha+3}+\frac{m}{(\alpha+2)(\alpha+3)}\left|f^{\prime \prime}\left(\frac{b^{\rho}}{m}\right)\right|^{q}\right)^{\frac{1}{q}}\right] ;
\end{aligned}
$$


3. if $\left|\mathrm{f}^{\prime \prime}\right|^{\mathrm{q}}$ is convex, i.e., $\mathrm{s}=\mathrm{m}=1$ and $\mu=0$, then we have the inequality

$$
\begin{aligned}
\mid \frac{\rho^{\alpha} \Gamma(\alpha+1)}{b^{\rho}-a^{\rho}} & {\left[\frac{b^{\rho}-x^{\rho}}{\left(x^{\rho}-a^{\rho}\right)^{\alpha}}{ }^{\rho} I_{x^{-}}^{\alpha} f\left(a^{\rho}\right)+\frac{x^{\rho}-a^{\rho}}{\left(b^{\rho}-x^{\rho}\right)^{\alpha}}{ }^{\rho} I_{x^{+}}^{\alpha} f\left(b^{\rho}\right)\right]-f\left(x^{\rho}\right) \mid } \\
\leqslant & \frac{\left(x^{\rho}-a^{\rho}\right)\left(b^{\rho}-x^{\rho}\right)}{(\alpha+1)\left(b^{\rho}-a^{\rho}\right)}\left(\frac{1}{\alpha+2}\right)^{1-\frac{1}{q}} \\
& \times\left[\left(x^{\rho}-a^{\rho}\right)\left(\frac{\left|f^{\prime \prime}\left(x^{\rho}\right)\right|^{q}}{\alpha+3}+\frac{\left|f^{\prime \prime}\left(a^{\rho}\right)\right|^{q}}{(\alpha+2)(\alpha+3)}\right)^{\frac{1}{q}}\right. \\
& \left.+\left(b^{\rho}-x^{\rho}\right)\left(\frac{\left|f^{\prime \prime}\left(x^{\rho}\right)\right|^{q}}{\alpha+3}+\frac{\left|f^{\prime \prime}\left(b^{\rho}\right)\right|^{q}}{(\alpha+2)(\alpha+3)}\right)^{\frac{1}{q}}\right] .
\end{aligned}
$$

Theorem 2.8. Let $\alpha, \rho>0$ and $f:[0, \infty) \rightarrow \mathbb{R}$ be a twice differentiable function on $\left(a^{\rho}, b^{\rho}\right)$ such that $f^{\prime \prime} \in$ $\mathrm{L}_{1}\left(\left[\mathrm{a}^{\rho}, \mathrm{b}^{\rho}\right]\right)$ where $0 \leqslant \mathrm{a}<\mathrm{b}$. If $\left|\mathrm{f}^{\prime \prime}\right|^{\mathrm{q}}$ is strongly $(\mathrm{s}, \mathrm{m})$-convex with modulus $\mu \geqslant 0$ (in the second sense) and $(\mathrm{s}, \mathrm{m}) \in(0,1]^{2}$, for $\mathrm{q}>1$, then for any $\mathrm{x} \in[\mathrm{a}, \mathrm{b}]$, the following inequality holds:

$$
\begin{aligned}
\mid \frac{\rho^{\alpha} \Gamma(\alpha+1)}{b^{\rho}-a^{\rho}} & {\left[\frac{b^{\rho}-x^{\rho}}{\left(x^{\rho}-a^{\rho}\right)^{\alpha}} I_{x^{-}}^{\alpha} f\left(a^{\rho}\right)+\frac{x^{\rho}-a^{\rho}}{\left(b^{\rho}-x^{\rho}\right)^{\alpha}} I_{x^{+}}^{\alpha} f\left(b^{\rho}\right)\right]-f\left(x^{\rho}\right) \mid } \\
\leqslant & \frac{\left(x^{\rho}-a^{\rho}\right)\left(b^{\rho}-x^{\rho}\right)}{(\alpha+1)\left(b^{\rho}-a^{\rho}\right)}\left(\frac{1}{r(\alpha+1)}\right)^{\frac{1}{r}} \\
& \times\left[\left(x^{\rho}-a^{\rho}\right)\left(\frac{\left|f^{\prime \prime}\left(x^{\rho}\right)\right|^{q}}{s+1}+\frac{m}{s+1}\left|f^{\prime \prime}\left(\frac{a^{\rho}}{m}\right)\right|^{q}-\frac{\mu}{6}\left(\frac{a^{\rho}}{m}-x^{\rho}\right)^{2}\right)^{\frac{1}{q}}\right. \\
& \left.+\left(b^{\rho}-x^{\rho}\right)\left(\frac{\left|f^{\prime \prime}\left(x^{\rho}\right)\right|^{q}}{s+1}+\frac{m}{s+1}\left|f^{\prime \prime}\left(\frac{b^{\rho}}{m}\right)\right|^{q}-\frac{\mu}{6}\left(\frac{b^{\rho}}{m}-x^{\rho}\right)^{2}\right)^{\frac{1}{q}}\right],
\end{aligned}
$$

where $\frac{1}{r}+\frac{1}{q}=1$

Proof. Using Lemma 2.1, the fact that $t^{\rho-1}=t^{\frac{\rho-1}{r}} t^{\frac{\rho-1}{q}}$, the Hölder's inequality and the strong $(s, m)-$ convexity of $\left|f^{\prime \prime}\right|^{q}$, we have

$$
\begin{aligned}
&\left|\frac{\rho^{\alpha} \Gamma(\alpha+1)}{b^{\rho}-a^{\rho}}\left[\frac{b^{\rho}-x^{\rho}}{\left(x^{\rho}-a^{\rho}\right)^{\alpha}} I_{x^{-}}^{\alpha} f\left(a^{\rho}\right)+\frac{x^{\rho}-a^{\rho}}{\left(b^{\rho}-x^{\rho}\right)^{\alpha}} I_{x^{+}}^{\alpha} f\left(b^{\rho}\right)\right]-f\left(x^{\rho}\right)\right| \\
& \leqslant \frac{\left(x^{\rho}-a^{\rho}\right)\left(b^{\rho}-x^{\rho}\right)}{(\alpha+1)\left(b^{\rho}-a^{\rho}\right)}\left[\rho\left(x^{\rho}-a^{\rho}\right) \int_{0}^{1} t^{\rho(\alpha+1} t^{\rho-1}\left|f^{\prime \prime}\left(t^{\rho} x^{\rho}+\left(1-t^{\rho}\right) a^{\rho}\right)\right| d t\right. \\
&\left.+\rho\left(b^{\rho}-x^{\rho}\right) \int_{0}^{1} t^{\rho(\alpha+1} t^{\rho-1}\left|f^{\prime \prime}\left(t^{\rho} x^{\rho}+\left(1-t^{\rho}\right) b^{\rho}\right)\right| d t\right] \\
& \leqslant \frac{\rho\left(x^{\rho}-a^{\rho}\right)\left(b^{\rho}-x^{\rho}\right)}{(\alpha+1)\left(b^{\rho}-a^{\rho}\right)}\left(\int_{0}^{1} t^{\rho r(\alpha+1)} t^{\rho-1} d t\right)^{\frac{1}{r}} \\
& \times\left[\left(x^{\rho}-a^{\rho}\right)\left(\int_{0}^{1} t^{\rho-1}\left|f^{\prime \prime}\left(t^{\rho} x^{\rho}+\left(1-t^{\rho}\right) a^{\rho}\right)\right|^{q} d t\right)^{\frac{1}{q}}\right. \\
&\left.+\left(b^{\rho}-x^{\rho}\right)\left(\int_{0}^{1} t^{\rho-1}\left|f^{\prime \prime}\left(t^{\rho} x^{\rho}+\left(1-t^{\rho}\right) b^{\rho}\right)\right| d t\right)^{\frac{1}{q}}\right] \\
& \leqslant \frac{\rho\left(x^{\rho}-a^{\rho}\right)\left(b^{\rho}-x^{\rho}\right)}{(\alpha+1)\left(b^{\rho}-a^{\rho}\right)}\left(\frac{1}{\rho r(\alpha+1)}\right)^{\frac{1}{r}}
\end{aligned}
$$




$$
\begin{aligned}
& \times\left[( x ^ { \rho } - a ^ { \rho } ) \left(\int _ { 0 } ^ { 1 } t ^ { \rho - 1 } \left(t^{s \rho}\left|f^{\prime \prime}\left(x^{\rho}\right)\right|^{q}+m\left(1-t^{\rho}\right)^{s}\left|f^{\prime \prime}\left(\frac{a^{\rho}}{m}\right)\right|^{q}\right.\right.\right. \\
& \left.\left.-\mu t^{\rho}\left(1-t^{\rho}\right)\left(\frac{a^{\rho}}{m}-x^{\rho}\right)^{2}\right) d t\right)^{\frac{1}{q}} \\
& +\left(b^{\rho}-x^{\rho}\right)\left(\int _ { 0 } ^ { 1 } t ^ { \rho - 1 } \left(t^{s \rho}\left|f^{\prime \prime}\left(x^{\rho}\right)\right|^{q}+m\left(1-t^{\rho}\right)^{s}\left|f^{\prime \prime}\left(\frac{b^{\rho}}{m}\right)\right|^{q}\right.\right. \\
& \left.\left.\left.-\mu t^{\rho}\left(1-t^{\rho}\right)\left(\frac{b^{\rho}}{m}-x^{\rho}\right)^{2}\right) d t\right)^{\frac{1}{q}}\right] \\
& =\frac{\rho\left(x^{\rho}-a^{\rho}\right)\left(b^{\rho}-x^{\rho}\right)}{(\alpha+1)\left(b^{\rho}-a^{\rho}\right)}\left(\frac{1}{\rho r(\alpha+1)}\right)^{\frac{1}{r}} \\
& \times\left[( x ^ { \rho } - a ^ { \rho } ) \left(\left|f^{\prime \prime}\left(x^{\rho}\right)\right|^{q} \int_{0}^{1} t^{s \rho+\rho-1} d t+m\left|f^{\prime \prime}\left(\frac{a^{\rho}}{m}\right)\right|^{q} \int_{0}^{1} t^{\rho-1}\left(1-t^{\rho}\right)^{s} d t\right.\right. \\
& \left.-\mu\left(\frac{a^{\rho}}{m}-x^{\rho}\right)^{2} \int_{0}^{1} t^{2 \rho-1}\left(1-t^{\rho}\right) d t\right)^{\frac{1}{q}} \\
& +\left(b^{\rho}-x^{\rho}\right)\left(\left|f^{\prime \prime}\left(x^{\rho}\right)\right|^{q} \int_{0}^{1} t^{s \rho+\rho-1} d t+m\left|f^{\prime \prime}\left(\frac{b^{\rho}}{m}\right)\right|^{q} \int_{0}^{1} t^{\rho-1}\left(1-t^{\rho}\right)^{s} d t\right. \\
& \left.\left.-\mu\left(\frac{b^{\rho}}{m}-x^{\rho}\right)^{2} \int_{0}^{1} t^{2 \rho-1}\left(1-t^{\rho}\right) d t\right)^{\frac{1}{q}}\right] \\
& =\frac{\rho\left(x^{\rho}-a^{\rho}\right)\left(b^{\rho}-x^{\rho}\right)}{(\alpha+1)\left(b^{\rho}-a^{\rho}\right)}\left(\frac{1}{\rho r(\alpha+1)}\right)^{\frac{1}{r}} \\
& \times\left[\left(x^{\rho}-a^{\rho}\right)\left(\frac{\left|f^{\prime \prime}\left(x^{\rho}\right)\right|^{q}}{\rho(s+1)}+\frac{m}{\rho(s+1)}\left|f^{\prime \prime}\left(\frac{a^{\rho}}{m}\right)\right|^{q}-\frac{\mu}{6 \rho}\left(\frac{a^{\rho}}{m}-x^{\rho}\right)^{2}\right)^{\frac{1}{q}}\right. \\
& +\left(b^{\rho}-x^{\rho}\right)\left(\frac{\left|f^{\prime \prime}\left(x^{\rho}\right)\right|^{q}}{\rho(s+1)}+\frac{m}{\rho(s+1)}\left|f^{\prime \prime}\left(\frac{b^{\rho}}{m}\right)\right|^{q}-\frac{\mu}{6 \rho}\left(\frac{b^{\rho}}{m}-x^{\rho}\right)^{\frac{1}{q}}\right] . \\
&
\end{aligned}
$$

This completes the proof.

Corollary 2.9. Under the conditions of Theorem 2.8,

1. if $\left|f^{\prime \prime}\right|^{q}$ is s-convex, i.e, $m=1$ and $\mu=0$, then we have the inequality

$$
\begin{aligned}
\mid \frac{\rho^{\alpha} \Gamma(\alpha+1)}{b^{\rho}-a^{\rho}} & {\left[\frac{b^{\rho}-x^{\rho}}{\left(x^{\rho}-a^{\rho}\right)^{\alpha}} I_{x^{-}}^{\alpha} f\left(a^{\rho}\right)+\frac{x^{\rho}-a^{\rho}}{\left(b^{\rho}-x^{\rho}\right)^{\alpha}} I_{x^{+}}^{\alpha} f\left(b^{\rho}\right)\right]-f\left(x^{\rho}\right) \mid } \\
\leqslant & \frac{\left(x^{\rho}-a^{\rho}\right)\left(b^{\rho}-x^{\rho}\right)}{(\alpha+1)\left(b^{\rho}-a^{\rho}\right)}\left(\frac{1}{r(\alpha+1)}\right)^{\frac{1}{r}} \\
& \times\left[\left(x^{\rho}-a^{\rho}\right)\left(\frac{\left|f^{\prime \prime}\left(x^{\rho}\right)\right|^{q}+\left|f^{\prime \prime}\left(a^{\rho}\right)\right|^{q}}{s+1}\right)^{\frac{1}{q}}\right. \\
& \left.+\left(b^{\rho}-x^{\rho}\right)\left(\frac{\left|f^{\prime \prime}\left(x^{\rho}\right)\right|^{q}+\left|f^{\prime \prime}\left(b^{\rho}\right)\right|^{q}}{s+1}\right)^{\frac{1}{q}}\right]
\end{aligned}
$$

2. if $\left|f^{\prime \prime}\right|^{q}$ is m-convex, i.e, $s=1$ and $\mu=0$, then we have the inequality

$$
\begin{gathered}
\left|\frac{\rho^{\alpha} \Gamma(\alpha+1)}{b^{\rho}-a^{\rho}}\left[\frac{b^{\rho}-x^{\rho}}{\left(x^{\rho}-a^{\rho}\right)^{\alpha}} I_{x^{-}}^{\alpha} f\left(a^{\rho}\right)+\frac{x^{\rho}-a^{\rho}}{\left(b^{\rho}-x^{\rho}\right)^{\alpha}}{ }^{\rho} I_{x^{+}}^{\alpha} f\left(b^{\rho}\right)\right]-f\left(x^{\rho}\right)\right| \\
\leqslant \frac{\left(x^{\rho}-a^{\rho}\right)\left(b^{\rho}-x^{\rho}\right)}{(\alpha+1)\left(b^{\rho}-a^{\rho}\right)}\left(\frac{1}{r(\alpha+1)}\right)^{\frac{1}{r}}
\end{gathered}
$$




$$
\begin{aligned}
& \times\left[\left(x^{\rho}-a^{\rho}\right)\left(\frac{\left|f^{\prime \prime}\left(x^{\rho}\right)\right|^{q}}{2}+\frac{m}{2}\left|f^{\prime \prime}\left(\frac{a^{\rho}}{m}\right)\right|^{q}\right)^{\frac{1}{q}}\right. \\
& \left.+\left(b^{\rho}-x^{\rho}\right)\left(\frac{\left|f^{\prime \prime}\left(x^{\rho}\right)\right|^{q}}{2}+\frac{m}{2}\left|f^{\prime \prime}\left(\frac{b^{\rho}}{m}\right)\right|^{q}\right)^{\frac{1}{q}}\right]
\end{aligned}
$$

3. if $\left|f^{\prime \prime}\right|^{\mathrm{q}}$ is convex, i.e, $\mathrm{s}=\mathrm{m}=1$ and $\mu=0$, then we have the inequality

$$
\begin{aligned}
\mid \frac{\rho^{\alpha} \Gamma(\alpha+1)}{b^{\rho}-a^{\rho}} & {\left[\frac{b^{\rho}-x^{\rho}}{\left(x^{\rho}-a^{\rho}\right)^{\alpha}} I_{x^{-}}^{\alpha} f\left(a^{\rho}\right)+\frac{x^{\rho}-a^{\rho}}{\left(b^{\rho}-x^{\rho}\right)^{\alpha}} I_{x^{+}}^{\alpha} f\left(b^{\rho}\right)\right]-f\left(x^{\rho}\right) \mid } \\
\leqslant & \frac{\left(x^{\rho}-a^{\rho}\right)\left(b^{\rho}-x^{\rho}\right)}{(\alpha+1)\left(b^{\rho}-a^{\rho}\right)}\left(\frac{1}{r(\alpha+1)}\right)^{\frac{1}{r}} \\
& \times\left[\left(x^{\rho}-a^{\rho}\right)\left(\frac{\left|f^{\prime \prime}\left(x^{\rho}\right)\right|^{q}+\left|f^{\prime \prime}\left(a^{\rho}\right)\right|^{q}}{2}\right)^{\frac{1}{q}}\right. \\
& \left.+\left(b^{\rho}-x^{\rho}\right)\left(\frac{\left|f^{\prime \prime}\left(x^{\rho}\right)\right|^{q}+\left|f^{\prime \prime}\left(b^{\rho}\right)\right|^{q}}{2}\right)^{\frac{1}{q}}\right] .
\end{aligned}
$$

\section{Conclusion}

We introduced several Ostrowski-type integral inequalities using the Katugampola fractional integral operators for functions whose second derivatives belongs to the Lebesgue $\mathrm{L}_{\mathrm{q}}$ spaces and also for functions whose second derivatives in absolute value at some powers are strongly $(\mathrm{s}, \mathrm{m})$-convex, in the second sense. Some particular cases have also been considered. Several other inequalities could be derived from by considering different values of the parameters in our results. In particular, if we take the limits as $\rho \rightarrow 1$ and $\rho \rightarrow 0^{+}$, then our results could be stated using the Riemann-Liouville and Hadamard fractional integrals respectively. The details are left for the interested reader.

\section{Acknowledgment}

The author wishes to express his profound gratitude to the anonymous referee for his/her valuable comments on the manuscript.

\section{References}

[1] M. Alomari, M. Darus, S. S. Dragomir, P. Cerone, Ostrowski type inequalities for the functions whose derivative are s-convex in second sense, Appl. Math. Lett., 23 (2010), 1071-1076. 1

[2] G. A. Anastassiou, Ostrowski type inequalities, Proc. Amer. Math. Soc., 123 (1995), 3775-3781. 1

[3] M. Bracamonte, J. Giménez, M. Vivas-Cortez, Hermite-Hadamard-Fejér type inequalities for strongly ( $\mathrm{s}, \mathrm{m})$-convex functions with modulus c, in second sense, Appl. Math. Inf. Sci., 10 (2016), 2045-2053. 1, 1.5, 1

[4] W. W. Breckner, Stetigkeitsaussagen für eine Klasse verallgemeinerter konvexer funktionen in topologischen linearen Raumen, Publ. Inst. Math. (Beograd) (N.S.), 23 (1978), 13-20. 1, 1.1, 1

[5] H. Chen, U. N. Katugampola, Hermite-Hadamard and Hermite-Hadamard-Fejr type inequalities for generalized fractional integrals, J. Math. Anal. Appl., 446 (2017), 1274-1291. 1

[6] S. S. Dragomir, A generalization of Ostrowski integral inequality for mappings whose derivatives belong to $\mathrm{L}_{1}[\mathrm{a}, \mathrm{b}]$ and applications in numerical integration, J. Comput. Anal. Appl., 3 (2001), 343-360. 1

[7] S. S. Dragomir, A generalization of the Ostrowski integral inequality for mappings whose derivatives belong to $\mathrm{L}_{\mathrm{p}}[\mathrm{a}, \mathrm{b}]$ and applications in numerical integration, J. Math. Anal. Appl., 255 (2001), 605-626.

[8] S. S. Dragomir, S. Wang, A new inequality of Ostrowski's type in $\mathrm{L}_{1}$-norm and applications to some special means and to some numerical quadrature rules, Tamkang J. Math., 28 (1997), 239-244.

[9] S. S. Dragomir, S. Wang, A new inequality of Ostrowskis type in $\mathrm{L}_{\mathrm{p}}$-norm, Indian J. Math., 40 (1998), 299-304. 1

[10] N. Eftekhari, Some remarks on (s, m)-convexity in the second sense, J. Math. Inequal., 8 (2014), 489-495. 1.3, 1

[11] G. Farid, U. N. Katugampola, M. Usman, Ostrowski type fractional integral inequalities for s-Godunova-Levin functions via Katugampola fractional integrals, Open J. Math. Sci., 1 (2017), 97-110. 1, 1 
[12] G. Farid, U. N. Katugampola, M. Usman, Ostrowski-type fractional integral inequalities for mappings whose derivatives are h-convex via Katugampola fractional integrals, Stud. Univ. Babeş-Bolyai Math., 63 (2018), 465-474. 1

[13] G. Farid, M. Usman, Ostrowski type k-fractional integral inequalities for MT-convex and h-convex functions, Nonlinear funct. Anal. Appl., 22 (2017), 627-639. 1

[14] U. N. Katugampola, New approach to a generalized fractional integral, Appl. Math. Comput., 218 (2011), 860-865. 1, $1.10,1.11,1$

[15] U. N. Katugampola, A new approach to generalized fractional derivatives, Bull. Math. Anal. Appl., 6 (2014), 1-15. 1

[16] B. Meftaha, K. Boukerrioua, Some new Ostrowski type inequalities for functions whose second derivative are h-convex via Riemann-Liouville fractional, Malaya J. Mat., 2 (2014), 445-459. 2.2

[17] A. M. Ostrowski, Über die Absolutabweichung einer differentiebaren Funktion von ihrem Integralmitelwert, Comment. Math. Helv., 10 (1938), 226-227. 1

[18] I. Podlubny, Fractional differential equations, Academic Press, San Diego, (1999). 1.6

[19] B. T. Polyak, Existence theorems and convergence of minimizing sequences in extremum problems with restrictions, Soviet Math. Dokl., 7 (1966), 72-75. 1, 1.4, 1

[20] S. G. Samko, A. A. Kilbas, O.I. Marichev, Fractional Integrals and Derivatives: Theory and applications, Gordon and Breach Science Publishers, Yverdon, (1993). 1.7

[21] E. Set, New inequalities of Ostrowski type for mappings whose derivatives are s-convex in the second sense via fractional integrals, Comput. Math. Appl., 63 (2012), 1147-1154. 1

[22] E. Set, I. Mumcu, Hermite-Hadamard Type Inequalities for Quasi-Convex Functions via Katugampola Fractional Integrals, Int. J. Anal. Appl., 16 (2018), 605-613. 1

[23] G. Toader, Some generalizations of the Convexity, Proc. Colloq. Approx. Optim. Cluj-Naploca (Cluj-Napoca, 1985), 1985 (1985), 329-338. 1, 1.2, 1 